\title{
Cross Modulation of Two Laser Beams at the Individual-Photon Level
}

\section{Citation}

Beck, Kristin M., Wenlan Chen, Qian Lin, Michael Gullans, Mikhail D. Lukin, and Vladan Vuletić. 2014. "Cross Modulation of Two Laser Beams at the Individual-Photon Level." Physical Review Letters 113 (11) (September). doi:10.1103/physrevlett.113.113603.

\section{Published Version}

doi:10.1103/PhysRevLett.113.113603

\section{Permanent link}

http://nrs.harvard.edu/urn-3:HUL.InstRepos:16914858

\section{Terms of Use}

This article was downloaded from Harvard University's DASH repository, and is made available under the terms and conditions applicable to Other Posted Material, as set forth at http:// nrs.harvard.edu/urn-3:HUL.InstRepos:dash.current.terms-of-use\#LAA

\section{Share Your Story}

The Harvard community has made this article openly available.

Please share how this access benefits you. Submit a story.

\section{Accessibility}




\title{
Cross Modulation of Two Laser Beams at the Individual-Photon Level
}

\author{
Kristin M. Beck, ${ }^{1}$ Wenlan Chen, ${ }^{1}$ Qian Lin, ${ }^{1}$ Michael Gullans, ${ }^{2,3}$ Mikhail D. Lukin, ${ }^{2}$ and Vladan Vuletic ${ }^{1}$ \\ ${ }^{1}$ Department of Physics and Research Laboratory of Electronics, Massachusetts Institute of Technology, Cambridge, \\ Massachusetts 02139, USA \\ ${ }^{2}$ Department of Physics, Harvard University, Cambridge, Massachusetts 02138, USA \\ ${ }^{3}$ Joint Quantum Institute, National Institute of Standards and Technology, Gaithersburg, Maryland 20899, USA
}

(Received 21 April 2014; published 12 September 2014)

\begin{abstract}
Deterministic photon-photon interactions are a long-standing goal in optical science. Using an atomic ensemble inside a cavity, we demonstrate the mutual cross modulation of two continuous light beams at the level of individual photons. The originally uncorrelated beams derived from independent lasers become anticorrelated, as evidenced by an equal-time cross-correlation function $g^{(2)}=0.89(1)$, showing that one photon in one beam extinguishes a photon in the other beam with a probability of $11(1) \%$. With further technical improvements, our approach should enable the nondestructive continuous detection of traveling optical photons.
\end{abstract}

DOI: 10.1103/PhysRevLett.113.113603

PACS numbers: 42.50.Pq, 42.50.Ct, 42.50.Gy

While, in vacuum, two light beams simply pass through one another, in nonlinear optical materials they can be made to interact. Even though, in standard bulk materials, the nonlinearity at the level of individual photons is very weak, techniques to enable strong photon-photon interactions are now actively explored. One approach to make two photons interact is to couple them strongly to the same atom, as can be achieved by means of a cavity [1-14]. Alternatively, photon-photon interactions can be mediated via interactions between two atoms that have absorbed the two photons, as can be achieved by means of electromagnetically induced transparency (EIT) [15] involving an atomic Rydberg state [16-21]. Recent advances in the optical domain using cavity quantum electrodynamics (QED) include a transistor gated by one stored photon [14], a nondestructive detector for single-photon pulses [22], a quantum phase switch [23], and a quantum phase gate between an atom and a photon [24]. Highlights using Rydberg excitation in a cold atomic ensemble include the dissipative filtering of photon number states [17], entanglement between a photon and a Rydberg excitation [18], microwave control of optical photons [19], and the observation of a bound state between two photons [20].

In this Letter, we demonstrate that two continuous light beams at different frequencies can modulate one another at the level of individual photons. This cross modulation is achieved by transmitting one of the beams through an atomic ensemble inside an optical cavity acting as a slowlight medium, such that the photons travel as slow-light polaritons $[15,25,26]$, whose atomic excitation component can block the transmission of another light beam through the cavity (see Fig. 1). The optical nonlinearity at the level of individual photons is evidenced by a cross-correlation function $g^{(2)}(0)=0.89(1)$, demonstrating continuous photon-photon switching with a probability of $11(1) \% . g^{(2)}(\tau)$ is asymmetric in the time separation $\tau$ between the photons in the two beams, revealing the different time scales for photon storage in the cavity and in the slow-light medium. Our analysis is in good agreement with the experimental results. This method may enable the continuous deterministic entanglement of two light beams for measurements below the standard quantum limit $[27,28]$, the nondestructive detection of individual photons by measuring cavity reflection instead of transmission [22], the imprinting of large photon-photon phase shifts [1,29] by conducting the same experiment away from atomic resonance, and the production of polarization entangled states by extension to another internal state [30,31].

The experimental system [14] is shown in Fig. 1(a). An ensemble of laser-cooled ${ }^{133} \mathrm{Cs}$ atoms is trapped inside an optical cavity. We choose a four-state $N$-type level structure $|f\rangle \leftrightarrow|d\rangle \leftrightarrow|c\rangle \leftrightarrow|e\rangle$, where the states $|f\rangle$ and $|c\rangle$ are stable ground states while $|d\rangle$ and $|e\rangle$ are electronic excited states. Two independent lasers drive the signal (free-space) mode $(|f\rangle \rightarrow|d\rangle)$ and the cavity mode $(|c\rangle \rightarrow|e\rangle)$. When these two transitions are connected by a coupling laser on the $|c\rangle \rightarrow|d\rangle$ transition, inducing EIT for the signal photons, this atomic structure mediates an effective interaction between the signal and cavity modes. In the absence of signal photons, the state $|c\rangle$ is unpopulated, and cavity photons incident onto and resonant with the empty cavity are simply transmitted. Without cavity photons, signal photons are transmitted through the ensemble, traveling in the medium as slow-light polaritons, a superposition of a photon and a collective atomic excitation to the state $|c\rangle$ $[15,25,26]$. Thus, photons arriving individually in either the signal or the cavity mode are transmitted through the system. However, simultaneously present photons in the two modes affect each other's transmission [14,26,29,33]: When a signal photon travels in the atomic ensemble as a 


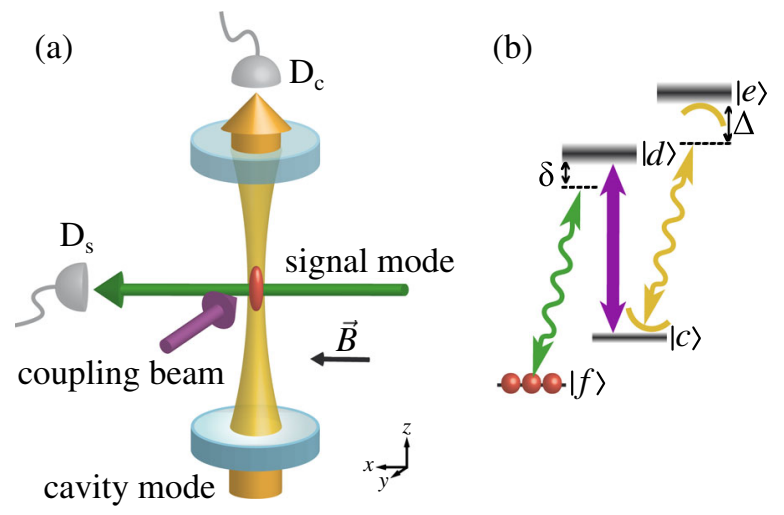

FIG. 1 (color online). Cross modulation of two laser beams at the level of individual photons. (a) Experimental setup and (b) atomic level scheme. An ensemble of laser-cooled atoms is trapped inside a high-finesse optical resonator and prepared in state $|f\rangle$. The ensemble and the coupling beam (resonant with the $|c\rangle \rightarrow|d\rangle$ transition) mediate interactions between signal $(|f\rangle \rightarrow|d\rangle)$ and cavity photons $(|c\rangle \rightarrow|e\rangle)$ : Signal photons travel through the medium as slow-light polaritons whose atomic excitation component in the state $|c\rangle$ blocks the transmission through the cavity. Photons are measured with photon counters $D_{s}$ and $D_{c}$.

slow-light polariton, the polariton's atomic component in state $|c\rangle$ reduces the cavity transmission by blocking the incident cavity photons and introducing additional cavity loss through photon scattering on the $|c\rangle \rightarrow|e\rangle$ transition. Conversely, the same scattering introduces loss and reduces the EIT transmission for the signal photon. If the coupling between the cavity and a single atom in state $|c\rangle$ is sufficiently strong, a cavity photon will block the transmission for a subsequently arriving signal photon, and vice versa.

To prepare a dense atomic ensemble, we overlap a magneto-optical trap with a far-off resonant optical-lattice trap of trap depth $U_{0} / h=6.9 \mathrm{MHz}$ operated at $937 \mathrm{~nm}$ in the $\mathrm{TEM}_{00}$ mode of the cavity. To maximize the number of atoms loaded into the optical-lattice trap, we compress the magneto-optical trap for $27 \mathrm{~ms}$ using a magnetic field gradient of $220 \mathrm{mT} / \mathrm{m}$ while cooling the atoms in an optical molasses. We thus typically load $5 \times 10^{5}$ atoms at $35 \mu \mathrm{K}$. To reduce cavity blocking and shifts due to atoms not addressed by the signal beam (which has a waist of $w_{f}=2.2 \mu \mathrm{m}$ ), we use the signal beam path to shelve atoms into the hyperfine manifold $F=3$ and then use a large beam on the $\left|6 S_{1 / 2}, F=4\right\rangle \rightarrow\left|6 P_{3 / 2}, F^{\prime}=5\right\rangle$ transition to remove unshelved atoms by radiation pressure. The remaining $N=2 \times 10^{4}$ atoms are then optically pumped into the state $|f\rangle \equiv\left|6 S_{1 / 2}, F=3, m_{F}=3\right\rangle$ along the quantization axis ( $x$ axis) defined by a $1.2 \mathrm{mT}$ magnetic field [Fig. 1(a)], resulting in a typical resonant optical depth $\mathcal{N}=0.9$ for the $\sigma^{+}$-polarized signal beam propagating along $x$. The $\pi$-polarized coupling beam is incident along the $y$ axis, with a variable Rabi frequency up to $\Omega /(2 \pi)=2 \mathrm{MHz}$. The four-state $N$-type level structure is $|f\rangle \leftrightarrow|d\rangle \leftrightarrow|c\rangle \leftrightarrow|e\rangle$, with two stable ground states $|f\rangle,|c\rangle \equiv\left|6 S_{1 / 2}, 4,4\right\rangle$ and two electronic excited states $|d\rangle \equiv$ $\left|6 P_{3 / 2}, 4,4\right\rangle$ and $|e\rangle \equiv\left|6 P_{3 / 2}, 5,5\right\rangle$. These states provide a good combination of oscillator strengths $f$ for both signal $\left(f_{f d}=0.42\right)$ and cavity $\left(f_{c e}=0.50\right)$ transitions. For the $|c\rangle \leftrightarrow|e\rangle$ transition, the single-atom cooperativity for an atom on the cavity axis (along $z$ ) at an antinode of the cavity standing wave is $\eta=4 g^{2} /(\kappa \Gamma)=4.3(2)[3,7,34]$, placing our system in the strong coupling limit $(\eta>1)$. Here, $2 g, \kappa$, and $\Gamma$ denote the single-photon Rabi frequency, cavity linewidth, and atomic linewidth, respectively, with values $2 g /(2 \pi)=1.79(5) \mathrm{MHz}, \kappa /(2 \pi)=142(1) \mathrm{kHz}$, and $\Gamma /(2 \pi)=5.22 \mathrm{MHz}$.

We first characterize how signal photons induce cavity blocking. For a lossless cavity in the absence of signal photons, the state $|c\rangle$ is unpopulated, so incident photons are perfectly transmitted through the cavity. (Our lossy cavity has a resonant transmission of $43 \%$, of which we detect $49 \%$.) When a signal photon travels in the atomic medium as a slow-light polariton, the polariton's atomic component in state $|c\rangle$ introduces additional cavity loss through photon scattering on the $|c\rangle \rightarrow|e\rangle$ transition. $n_{a}$ atoms in state $|c\rangle$ reduce cavity transmission by a factor $\left(1+n_{a} \eta\right)^{-2}$ [34]. Since we use continuous beams, the actual transmission depends on the average atomic population $\left\langle n_{a}\right\rangle \approx R_{s}\left(1-e^{-\mathcal{N}}\right) \Gamma / \Omega^{2}$, where $R_{s}$ is the incident signal rate, $\Gamma / \Omega^{2}$ is the EIT lifetime, and $\mathcal{N}$ is the optical depth. In Fig. 2, the measured cavity transmission is displayed versus signal photon number, showing good agreement with the predicted transmission, for a spatially averaged cooperativity of $\eta=1.2$ and $\left\langle n_{s}\right\rangle=1$.

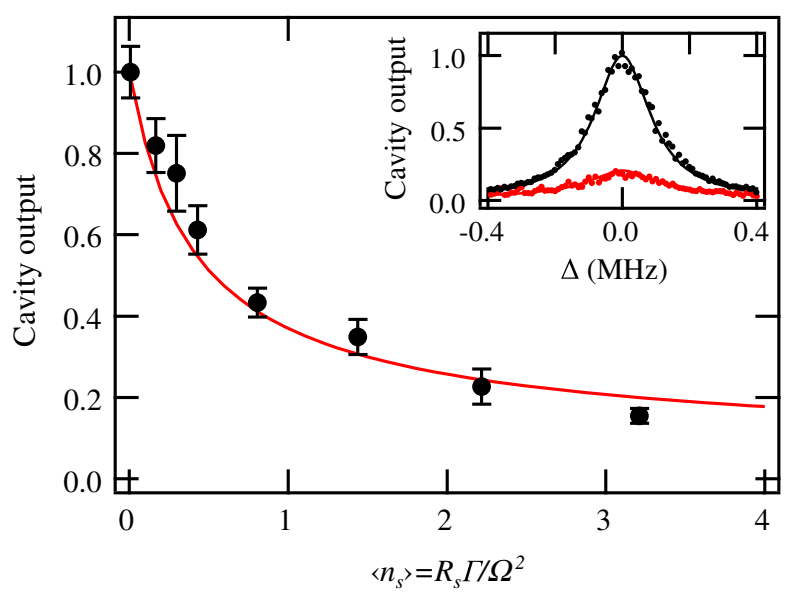

FIG. 2 (color online). Switching of cavity photons by signal light. Fractional cavity transmission is plotted versus average signal photon number per EIT lifetime $\left\langle n_{s}\right\rangle=R_{s} \Gamma / \Omega^{2}$. The measurement was performed with $\left.\left\langle n_{c}\right\rangle\right|_{n_{s}=0}=2.7$ and $\Omega^{2} / \Gamma=$ 1.4(1) $\mu \mathrm{s}^{-1}$. The expected transmission (solid curve) is the calculated cavity blocking (see the text). Inset: Illustration of cavity transmission reduction (red curve) due to signal photons as a function of light cavity detuning $\Delta$. Error bars are \pm 1 s.d. 
While signal photons induce cavity loss, cavity photons reduce the transmission of the signal beam. The transmission for resonant EIT is $T_{s}=\exp \left[-\mathcal{N} /\left(1+\Omega^{2} /(\Gamma \gamma)\right)\right]$; i.e., the optical depth $\mathcal{N}$ is reduced by the factor $1+\Omega^{2} /(\Gamma \gamma)$ due to EIT [15,25]. Here, $\gamma$ is the decoherence between the two ground states $|f\rangle$ and $|c\rangle$, which consists of two components: the thermal Doppler broadening characterized by a Gaussian profile of full width at half maximum (FWHM) $2 \gamma_{d}$ and the decoherence $\gamma_{c}$ induced by cavitylight coupling $|c\rangle$ to the unstable excited state $|e\rangle$. The expected cavity-light-induced decoherence for $n_{c}$ cavity photons is $\gamma_{c}=4 n_{c} g^{2} / \Gamma=n_{c} \eta \kappa$. When the EIT lifetime is much less than the cavity lifetime, the intracavity photon number is simply the average intracavity photon number without signal photons $\left.\left\langle n_{c}\right\rangle\right|_{n_{s}=0}$. However, cavity blocking by the polariton component in state $|c\rangle$ reduces the intracavity photon number when $\Gamma / \Omega^{2} \gtrsim \kappa^{-1}$, such that $n_{c}=$ $\left.\left\langle n_{c}\right\rangle\right|_{n_{\mathrm{s}}=0} /(1+\tilde{\eta})^{2}$ with $\tilde{\eta}=\eta /\left(1+\Omega^{2} /(\kappa \Gamma)\right)$ in the limit of $\mathcal{N}^{s}>1$.

EIT spectra taken with different intracavity photon numbers are fit to determine the decoherence rate $\gamma_{c}$ as a function of $\left.\left\langle n_{c}\right\rangle\right|_{n_{s}=0}=R_{c} /[(\kappa / 2)(\mathcal{T} /(\mathcal{T}+\mathcal{L}))]$, as shown in Fig. 3. Here, $R_{c}$ is the photon rate transmitted through the cavity in the absence of atoms in $|c\rangle, \mathcal{T}=$ $2.7 \times 10^{-5}$ is the mirror transmission, and $\mathcal{L}=1.4 \times 10^{-5}$ is the mirror loss. We approximate the underlying convolution of a Gaussian profile with FWHM $2 \gamma_{d}$ and Lorentzian profile of linewidth $\gamma_{c}$ by a Lorentzian profile of linewidth $\gamma$. From an approximation to the width of the Voigt profile, accurate to better than $1 \%$ [35], we expect $\gamma=0.535 \gamma_{c}+\sqrt{0.217 \gamma_{c}^{2}+\gamma_{d}^{2}}$. The fit agrees well with the data, giving $\gamma_{d} /(2 \pi)=110(10) \mathrm{kHz}$ for the Doppler broadening and a cavity-induced decoherence per photon of $\gamma_{c} /\left(2 \pi n_{c}\right)=70(25) \mathrm{kHz}$. This value is in excellent agreement with the expected value $\gamma_{c} /\left(2 \pi n_{c}\right)=71(8) \mathrm{kHz}$.

The above measurements demonstrate that the signal and cavity light attenuate each other at the level expected from cavity QED calculations. To present direct evidence for photon-photon interactions, we measure the second-order cross-correlation function $g^{(2)}(\tau)=\left\langle n_{c}(0) n_{s}(\tau)\right\rangle /\left(\left\langle n_{c}(0)\right\rangle\right.$ $\left.\left\langle n_{s}(\tau)\right\rangle\right)$ for weak coherent states with $\left\langle n_{c}\right\rangle,\left\langle n_{s}\right\rangle \ll 1$ incident in both the signal and cavity modes. Here, $\tau=$ $t_{s}-t_{c}$ is the time separation between the signal and cavity photons. In this limit, the cross-correlation function can be interpreted as the modified transmission of one mode conditioned on the presence of a photon in the other mode. A calculation shows that $g^{(2)}(\tau)$ is given by (see the Supplemental Material [31])

$$
g^{(2)}(\tau)=\left[1-\left(1-e^{-\mathcal{N} /(2 \zeta)}\right) \frac{\eta}{1+\eta} e^{-\kappa_{\gtrless}|\tau| / 2}\right]^{2} .
$$

For $\tau<0, \kappa_{<}=\kappa$ is the cavity linewidth, and, for $\tau>0$, $\kappa_{>}=\Omega^{2} / \Gamma+\gamma_{d}$ is the EIT linewidth including decoherence
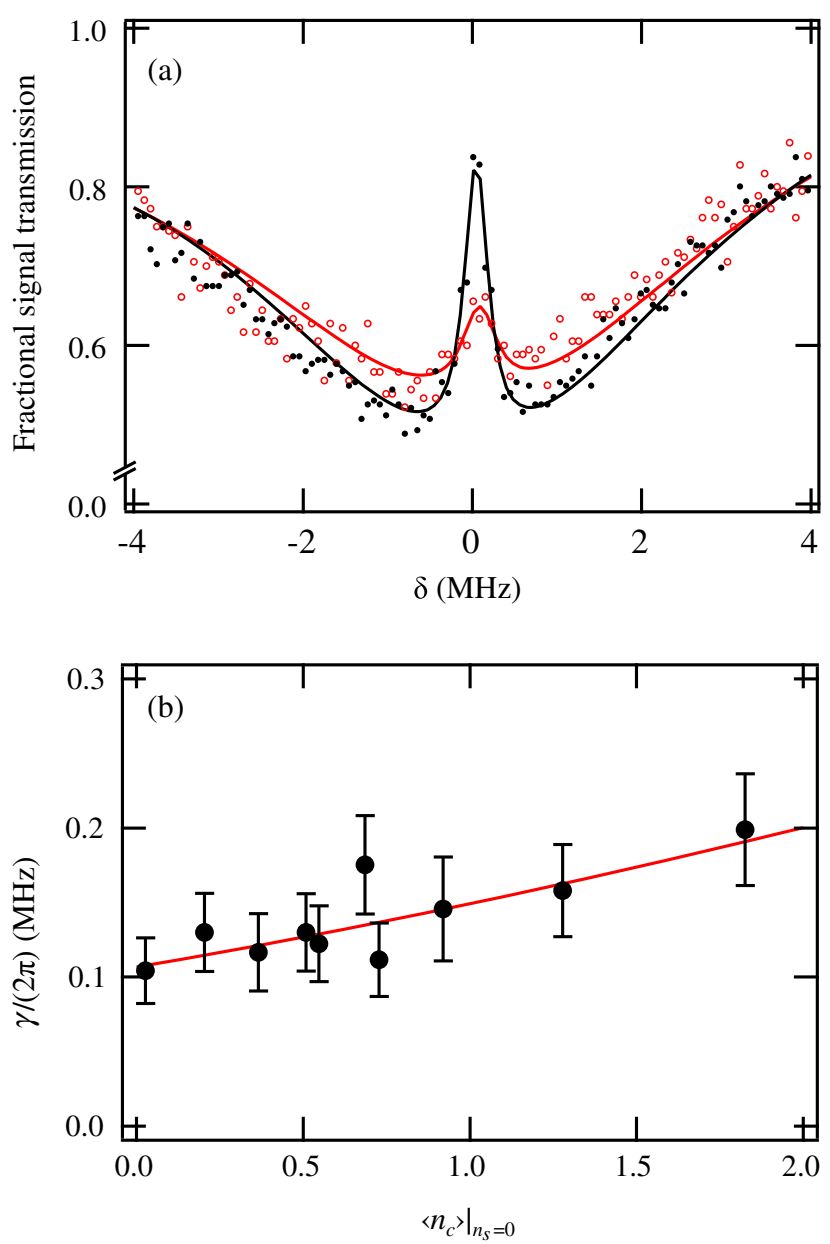

FIG. 3 (color online). Switching of signal photons by cavity light. (a) Representative EIT spectra as a function of signal detuning for $\left.\left\langle n_{c}\right\rangle\right|_{n_{\mathrm{s}}=0}=0$ (black curve, high transmission) and 11.5 (red curve, low transmission) cavity photons. (b) EIT decoherence rates are plotted versus cavity photon number for $\Omega^{2} / \Gamma=1.4(1) \mu \mathrm{s}^{-1}$. A fit (solid line) gives a cavity-lightinduced decoherence rate of $\gamma_{c} /\left(2 \pi n_{c}\right)=70(25) \mathrm{kHz}$. Error bars are \pm 1 s.d.

$\gamma_{d}$ [15]. In the correction factor $\zeta=\kappa_{>} /\left(\kappa_{>}-\gamma\right)\left[1+\kappa_{>} /\right.$ $\left.\left(\kappa_{<}(1+\eta)\right)\right]>1$, the first multiplicative factor arises from imperfect EIT, which reduces the cross modulation by decreasing the lifetime of the polariton. The second multiplicative factor accounts for the difference in the full (broadened) cavity lifetime and the polariton lifetime: when the polariton lifetime is shorter than the cavity lifetime $\left(\kappa_{>}>\kappa_{<}\right)$, the interaction time (and thus the cross modulation) is reduced.

In the limit of large optical depth $\mathcal{N} / \zeta \gg \ln \eta$, every incoming free-space photon is converted into a slow-light polariton [15,25] with a near-unity atomic excitation component in state $|c\rangle$. In this case, the cavity transmission is modified by the single intracavity atom [34] and is given by $(1+\eta)^{-2}$, which is also the minimum value of the crosscorrelation function $g^{(2)}(0)$ in this limit. In the opposite limit of large cooperativity $\eta \gg 1$ and moderate optical 
depth $\mathcal{N} / \zeta \ll \ln \eta$, the cavity photon completely destroys EIT, and $g^{(2)}(0) \approx e^{-\mathcal{N}}$ is simply the probability for the free-space photon to pass through the absorbing medium in the absence of EIT. Interestingly, the correlation function $g^{(2)}(\tau)$ is asymmetric in the time separation $\tau$ between the photons [4]. This can be understood as follows: the detection of a cavity photon at time $t=0$ implies that the EIT transmission must have been reduced for times $t<0$ on a time scale on the order of the cavity lifetime $\kappa^{-1}$ and will approach its uncorrelated steady-state value $g^{(2)}(\infty)=1$ for times $t>0$ with a time constant determined by the polariton lifetime, or equivalently, the EIT linewidth $\kappa_{>}=\Omega^{2} / \Gamma+\gamma_{d}$. An analogous argument can be made if one assumes the free-space photon to be detected at $t=0$.

The measured cross-correlation functions $g^{(2)}(\tau)$ displayed in Fig. 4 show that photons in the two modes are uncorrelated for large time separation $\tau$ but display a marked anticorrelation dip near $\tau=0$ : when the two photons derived from independent lasers arrive nearly simultaneously, they reduce each other's transmission through the system. The data in Figs. 4(a)-4(c) are well described by Eq. (1) using a three-parameter fit with the zero-time value $g^{(2)}(0)$ and the two decay rate constants $\kappa_{\gtrless}$. The time constants $\kappa_{\gtrless}$ obtained from the fit confirm the asymmetric shape of the cross-correlation function [Fig. 4(d)]: the negative-time constant $\kappa_{<}=1.6(1) \mu \mathrm{s}^{-1}$ is independent of coupling Rabi frequency and $80 \%$ larger than the cavity linewidth $\kappa$, presumably due to the occasional presence of absorbing atoms in the state $|c\rangle$ due to imperfect optical pumping. (Note that even only one atom out of $N=2 \times 10^{4}$ is sufficient to substantially increase the cavity linewidth by a factor of $1+\eta$.) The positive-time constant $\kappa_{>}$is linearly dependent on coupling beam intensity. A fit to $a \Omega^{2} / \Gamma+b$ gives a slope $a=0.7(1)$ and the $y$-axis intercept $b=1.2(2) \mu \mathrm{s}^{-1}$. These are in reasonable agreement with the expected slope $a=1$ and the decoherence rate $b=\gamma=0.69(6) \mu \mathrm{s}^{-1}$ extracted from the EIT curves in Fig. 3. The measured $g^{(2)}(0)$ is between 0.89 (1) and 0.91(1) for the three values of the coupling Rabi frequency. This agrees well with the prediction from Eq. (1) when we average over the spatial variation of the cooperativity, yielding values between 0.90(1) and 0.93(1), using the independently measured optical depth, fitted $\kappa_{\gtrless}$ and $\gamma$, and a reduced cooperativity $\eta^{\prime}=\eta\left(\kappa / \kappa_{<}\right)$due to imperfect optical pumping. The measured zero-time correlation $g^{(2)}(0)$ corresponds to a photon-photon modulation efficiency of $1-g^{(2)}(0)=0.11(1)$ of one photon by the other in continuous operation.

Future realistic technical improvements can further increase this modulation efficiency. We have already operated the system with an optical depth as high as 1.5, which could be further increased by Bose condensing the sample. By reorienting the coupling beam to a
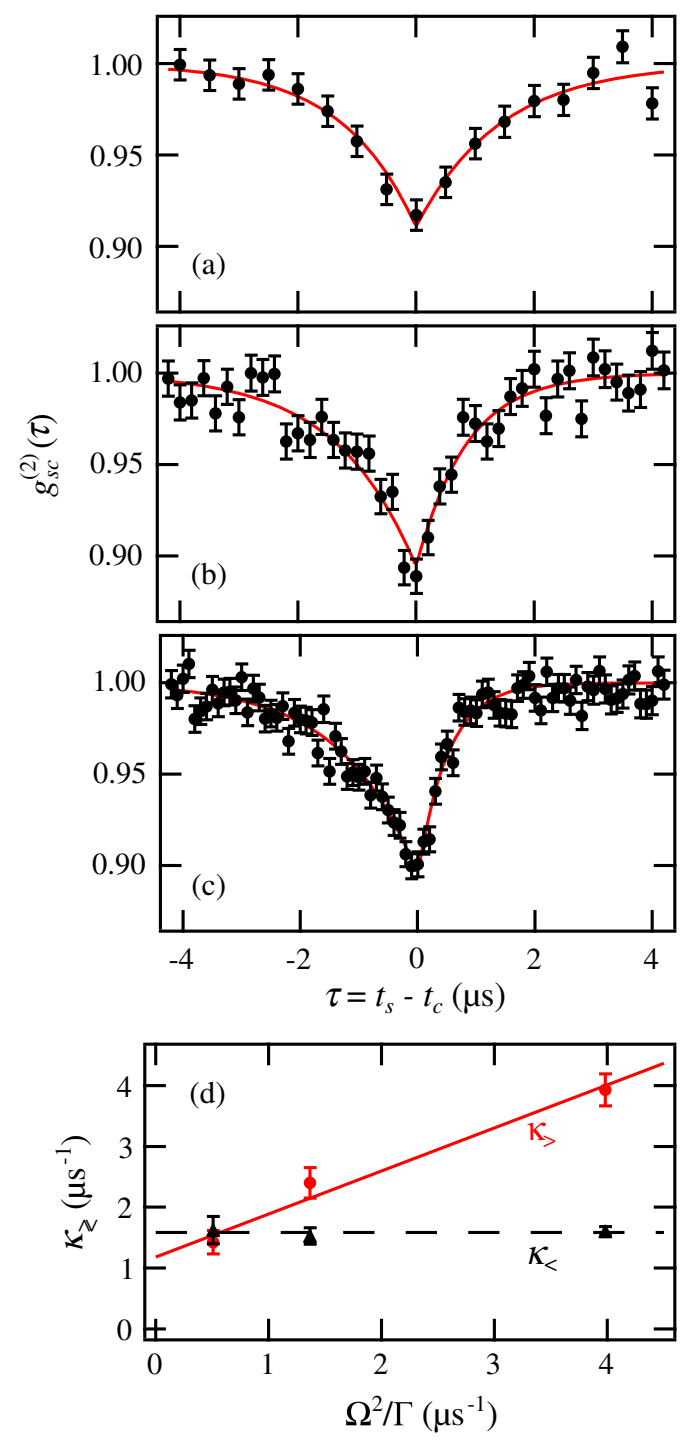

FIG. 4 (color online). Mutual photon-photon interaction between continuous laser beams. The second-order cross-correlation function $g^{(2)}(\tau)$ versus time separation $\tau=t_{s}-t_{c}$ for different coupling beam powers: $\Omega^{2} / \Gamma=$ (a) $0.5(1) \mu \mathrm{s}^{-1}$, (b) $1.4(1) \mu \mathrm{s}^{-1}$, and (c) $4.0(1) \mu \mathrm{s}^{-1}$. Fits of the data to the model (see the text) yield (a) $\left\{g^{(2)}(0), \kappa_{<} / \mu \mathrm{s}^{-1}, \kappa_{>} / \mu \mathrm{s}^{-1}\right\}=\{0.91(1), 1.6(2), 1.4(2)\}$, (b) $\{0.89(1), 1.5(1), 2.4(3)\}$, and (c) $\{0.90(1), 1.6(1), 3.9(3)\}$. (d) Fitted rate constants $\kappa_{\gtrless}$ versus the EIT linewidth $\Omega^{2} / \Gamma$. The positive-time rate constant fits to $\kappa_{>}=a \Omega^{2} / \Gamma+b$ with slope $a=$ $0.7(1)$ and $y$-axis intercept $b=1.2(2) \mu \mathrm{s}^{-1}$. The negative-time rate constant $\kappa_{<}=1.6(1) \mu \mathrm{s}^{-1}$ is independent of $\Omega^{2}$. Here, $\Omega^{2} / \Gamma$ was independently determined from separately measured EIT spectra. The measurements were performed at photon numbers $\left\langle n_{c}\right\rangle \approx 0.2$ and $\left\langle n_{s}\right\rangle \approx 0.2$ when integrated over time windows $1 / \kappa_{<}$and $1 / \kappa_{>}$, respectively.

Doppler-free configuration, we have measured $\gamma_{d}=10 \mathrm{kHz}$. We have achieved within our group cavities with antinode cooperativity exceeding 20 using small radius of curvature mirrors [36]. For a single system that simultaneously achieves $\eta=20, \mathcal{N}=3$, and $\gamma_{d}=10 \mathrm{kHz}$ with atoms 
confined to antinodes of the cavity coupling, the expected photon-photon modulation efficiency is $1-g^{(2)}(0)=0.91$.

We acknowledge partial support from NSF, CUA, the AFOSR Quantum Memories MURI, and the ARO QuISM MURI. K. M. B. acknowledges support from NSF GRFP under Grant No. 0645960. M. G. acknowledges helpful discussions with M. Hafezi.

[1] Q. A. Turchette, C. J. Hood, W. Lange, H. Mabuchi, and H. J. Kimble, Phys. Rev. Lett. 75, 4710 (1995).

[2] P. Michler, A. Kiraz, C. Becher, W. V. Schoenfeld, P. M. Petroff, L. Zhang, E. Hu, and A. Imamoglu, Science 290, 2282 (2000).

[3] K. M. Birnbaum, A. Boca, R. Miller, A. D. Boozer, T. E. Northup, and H. J. Kimble, Nature (London) 436, 87 (2005).

[4] K. Hennessy, A. Badolato, M. Winger, D. Gerace, M. Atatüre, S. Gulde, S. Fält, E. L. Hu, and A. Imamoğlu, Nature (London) 445, 896 (2007).

[5] Y. Colombe, T. Steinmetz, G. Dubois, F. Linke, D. Hunger, and J. Reichel, Nature (London) 450, 272 (2007).

[6] F. Brennecke, T. Donner, S. Ritter, T. Bourdel, M. Köhl, and T. Esslinger, Nature (London) 450, 268 (2007).

[7] A. Kubanek, A. Ourjoumtsev, I. Schuster, M. Koch, P. W. H. Pinkse, K. Murr, and G. Rempe, Phys. Rev. Lett. 101, 203602 (2008).

[8] I. Fushman, D. Englund, A. Faraon, N. Stoltz, P. Petroff, and J. Vučković, Science 320, 769 (2008).

[9] H. Tanji-Suzuki, W. Chen, R. Landig, J. Simon, and V. Vuletić, Science 333, 1266 (2011).

[10] C. Lang, D. Bozyigit, C. Eichler, L. Steffen, J. M. Fink, A. A. Abdumalikov, M. Baur, S. Filipp, M. P. da Silva, A. Blais, and A. Wallraff, Phys. Rev. Lett. 106, 243601 (2011).

[11] D. Englund, A. Majumdar, M. Bajcsy, A. Faraon, P. Petroff, and J. Vučković, Phys. Rev. Lett. 108, 093604 (2012).

[12] R. Bose, D. Sridharan, H. Kim, G. S. Solomon, and E. Waks, Phys. Rev. Lett. 108, 227402 (2012).

[13] T. Volz, A. Reinhard, M. Winger, A. Badolato, K. J. Hennessy, E. L. Hu, and A. Imamoğlu, Nat. Photonics 6, 605 (2012).

[14] W. Chen, K. M. Beck, R. Bücker, M. Gullans, M. D. Lukin, H. Tanji-Suzuki, and V. Vuletić, Science 341, 768 (2013).

[15] M. Fleischhauer, A. Imamoglu, and J. P. Marangos, Rev. Mod. Phys. 77, 633 (2005).

[16] Y. O. Dudin and A. Kuzmich, Science 336, 887 (2012).
[17] T. Peyronel, O. Firstenberg, Q.-Y. Liang, S. Hofferberth, A. V. Gorshkov, T. Pohl, M. D. Lukin, and V. Vuletić, Nature (London) 488, 57 (2012).

[18] L. Li, Y. O. Dudin, and A. Kuzmich, Nature (London) 498, 466 (2013).

[19] D. Maxwell, D. J. Szwer, D. Paredes-Barato, H. Busche, J. D. Pritchard, A. Gauguet, K. J. Weatherill, M. P. A. Jones, and C. S. Adams, Phys. Rev. Lett. 110, 103001 (2013).

[20] O. Firstenberg, T. Peyronel, Q.-Y. Liang, A. V. Gorshkov, M. D. Lukin, and V. Vuletić, Nature (London) 502, 71 (2013).

[21] J. D. Pritchard, D. Maxwell, A. Gauguet, K. J. Weatherill, M. P. A. Jones, and C. S. Adams, Phys. Rev. Lett. 105, 193603 (2010).

[22] A. Reiserer, S. Ritter, and G. Rempe, Science 342, 1349 (2013)

[23] T. G. Tiecke, J. D. Thompson, N. P. de Leon, R. L. Liu, V. Vuletić, and M. D. Lukin, Nature (London) 508, 241 (2014).

[24] A. Reiserer, N. Kalb, G. Rempe, and S. Ritter, Nature (London) 508, 237 (2014).

[25] S. E. Harris, Phys. Today 50, No. 7, 36 (1997).

[26] S. E. Harris and Y. Yamamoto, Phys. Rev. Lett. 81, 3611 (1998).

[27] J. J. Cooper, D. W. Hallwood, and J. A. Dunningham, Phys. Rev. A 81, 043624 (2010).

[28] J. Joo, W. J. Munro, and T. P. Spiller, Phys. Rev. Lett. 107, 083601 (2011).

[29] H. Schmidt and A. Imamoğlu, Opt. Lett. 21, 1936 (1996).

[30] H. Tanji, J. Simon, S. Ghosh, B. Bloom, and V. Vuletić, Phys. Scr. T135, 014010 (2009).

[31] See Supplemental Material athttp://link.aps.org/supplemental/ 10.1103/PhysRevLett.113.113603, which includes Ref. [32], for the derivation of Eq. (1) and an extension of our scheme to produce polarization entangled states.

[32] H. J. Carmichael, R. J. Brecha, and P. R. Rice, Opt. Commun. 82, 73 (1991).

[33] A. Imamoglu, H. Schmidt, G. Woods, and M. Deutsch, Phys. Rev. Lett. 79, 1467 (1997).

[34] H. Tanji-Suzuki, I. D. Leroux, M. H. Schleier-Smith, M. Cetina, A. T. Grier, J. Simon, and V. Vuletić, Adv. At. Mol. Opt. Phys. 60, 201 (2011).

[35] J. J. Olivero and R. L. Longbothum, J. Quant. Spectrosc. Radiat. Transfer 17, 233 (1977).

[36] D. Hunger, T. Steinmetz, Y. Colombe, C. Deutsch, T. W. Hänsch, and J. Reichel, New J. Phys. 12, 065038 (2010). 